\title{
Endovascular Rescue Treatment for Occlusion of Renal Arteries Resulting in Acute Renal Failure
}

\author{
Pawel Latacz $^{1}$, Marian Simka ${ }^{2 *}$, Marcin Krzanowski ${ }^{1}$, Joanna Dolipska ${ }^{1}$, Wladysław Sułowicz ${ }^{1}$ \\ ${ }^{1}$ Jagiellonian University Collegium Medicum, Kraków, Poland \\ ${ }^{2}$ University of Opole, Department of Anatomy, Opole, Poland
}

*Corresponding Author: Marian Simka, University of Opole, Department of Anatomy, Opole, Poland, Email: mariansimka@poczta.onet.pl

\begin{abstract}
Urgent revascularization of renal arteries for their occlusions resulting in acute renal failure still remains a therapeutic challenge. In this paper we describe 3 cases of successful endovascular interventions for acute non-iatrogenic occlusions of renal arteries. In the first patient we managed thrombotic occlusion of the renal artery that coexisted with gastrointestinal bleeding. We recanalized occluded renal artery using an extraction catheter and stenting, and managed the bleeding with the use of embolization coils. Endovascular repair resulted in normal dieresis, still several days after the intervention gastrointestinal hemorrhage recurred and patient died due to multiple organ failure. In the second patient there was acute renal failure of the solitary kidney, which resulted from occlusion of the renal artery 4 months after endovascular repair of abdominal aortic aneurysm. We performed thrombectomy of the renal artery and implanted covered stent. In the third patient an acute occlusion of renal artery coexisted with occlusion of distal abdominal aorta. We revascularized the renal artery, superior mesenteric artery and aorta with stentgrafts, using the chimney technique. In the second and third patient the treatment resulted in restored diuresis and normal flow through repaired renal artery at 6-months follow up.
\end{abstract}

Keywords: angioplasty, kidney failure, renal artery obstruction, stents, thrombectomy

\section{INTRODUCTION}

Urgent revascularization of the renal artery in a case of its acute obstruction that results in renal failure still remains a therapeutic challenge. Ischemia of the kidneys is often asymptomatic and it is also difficult to determine when a compromised renal circulation results in irreversible damage of the organ. In addition, this problem usually affects severely sick patients presenting with significant comorbidities. Renal artery angioplasty for the treatment of acute renal ischemia is primarily performed as urgent management of iatrogenic occlusion of this artery during implantation of stent grafts in the abdominal aorta, especially when the so-called chimney technique is used. Here we present three cases of such an endovascular repair for acute renal ischemia, which were not caused by intraoperative occlusion of renal arteries.

\section{CASE PRESENTATIONS}

\subsection{Patient1}

An 85-year-old female patient was admitted to

ARC Journal of Cardiology our hospital because of severe gastrointestinal bleeding. She presented with grade 3 arterial hypertension, profound dislipidemia and morbid obesity. Since endoscopic examination of the stomach and duodenum failed to reveal source of bleeding and there was still bleeding despite pharmacological treatment, she underwent exploratory laparotomy. Inspection of the abdominal cavity and palpation of the intestines did not reveal an obvious abnormality, but unexpectedly there was retroperitoneal hematoma. A source of bleeding into this hematoma was not found. Considering overall poor clinical status of the patient, which deteriorated during the operation, packing of the hematoma with several large abdominal packs was performed. It was not possible to perform further exploration to find the source of gastrointestinal bleeding. Patient was transferred to the intensive care unit, where her clinical status, although severe, slightly improved.

However, on the $6^{\text {th }}$ day after laparotomy there were laboratory signs of acute renal failure (serum creatinine $>300 \mu \mathrm{mol} / \mathrm{L}$, urea $>12$ 
$\mathrm{mmol} / \mathrm{L}$, potassium $6.7 \mathrm{mmol} / \mathrm{L})$, which required urgent hemodialysis. Clinical status of the patient again deteriorated. Since it was suspected that renal failure resulted from ischemia of the kidneys, perhaps caused by abdominal packing, and there was still an active gastrointestinal bleeding, CT angiography aimed at exploration of both pathologies was performed. This imaging examination revealed occlusion of the right renal artery and features of nephrosclerosis of the right kidney, and also critical thrombotic stenosis of the left renal artery. There were also radiographic signs of bleeding into the duodenum. The patient was consulted be nephrologists, who-in spite of probable long time of occlusion of renal arteries-recommended an attempt of endovascular revascularisation of the left kidney and stopping gastrointestinal bleeding.

\subsubsection{Intervention}

During endovascular intervention patient was unstable, she required mechanical ventilation and continuous intravenous infusion of dopamine. With the use of $6 \mathrm{~F} / 45 \mathrm{~cm}$ Destination $^{\circledR}$ guiding sheath (Terumo, Tokyo, Japan) and 5F RBI hydrophilic diagnostic catheter Impress $^{\circledR}$ (Merit Medical Systems Inc., South Jordan, UT, USA) we navigated through subtotal occlusion of the left renal artery. Then, over V-14 Control Wire TM (Boston Scientific, Natick, MA, USA) hydrophilic-coated guidewire we introduced extraction catheter Hunter $^{\circledR}$ (IHT Cordynamic, Barcelona, Spain). With the use of this device we removed thrombi from the renal artery and also to remove remaining small emboli occluding side branches. Since control angiography still demonstrated about $90 \%$ stenosis of proximal part of the left renal artery, we implanted 4.5/15 mm Renal Dynamic stent (Biotronik, Berlin, Germany), which was expanded using balloon inflation under pressure of 8 atm for $30 \mathrm{~s}$. After stent implantation there was good flow of contrast to renal parenchyma. Then we performed angiographic inspection of intestinal arteries. We found extravasation to the duodenum from the branch of the inferior pancreaticoduodenal artery. We introduced telescopically into this branch microcatheter Progreat ${ }^{\circledR}$ (Terumo, Tokyo, Japan) and deployed two Tornado ${ }^{\circledR}$ (Cook Medical, Bloomington, IN, US A) embolization coils, which fully controlled bleeding. After endovascular procedure patient returned to the intensive care unit. On the next day the revascularized kidney started to produce urine, and from $4^{\text {th }}$ postprocedural day its function improved enough to stop hemodialysis. However, on $10^{\text {th }}$ postprocedural day the gastrointestinal bleeding recurred. All attempts to stop the hemorrhage failed and the patients died due to multiple organ failure.

\subsection{Patient2}

A 60-year-old male patient, who 4 months before underwent endovascular repair of abdominal aortic aneurysm with the Nellix ${ }^{\circledR}$ EndoVascular Aneurysm Sealing System (Endologix, Irvine, CA, USA). This patient was admitted to the hospital due to acute anuria. He presented with biochemical signs of renal failure (serum creatinine: $300 \square \mathrm{mol} / \mathrm{L}$, potassium 6.5 $\mathrm{mmol} / \mathrm{L}$ ) and required hemodialysis. Catheter angiography revealed occlusion of the right renal artery, which provided blood supply to the solitary right kidney. Origin of this occluded artery was situated at the upper edge of aortic stentgraft.

\subsubsection{Intervention}

Through the brachial access, using the $6 \mathrm{~F} / 90 \mathrm{~cm}$ Destination ${ }^{\circledR}$ guiding sheath (Terumo, Tokyo, Japan) and 5F Vertebral hydrophilic diagnostic catheter Impress ${ }^{\circledR}$ (Merit Medical Systems Inc., South Jordan, UT, USA) we cannulated the right renal artery. Then we navigated through occluded segment of this artery using the 300 $\mathrm{cm}$ long V-14 ControlWireTM (Boston Scientific, Natick, MA, USA) guidewire and introduced the extraction catheter Hunter ${ }^{\circledR}$ (IHT Cordynamic, Barcelona, Spain). However, recanalization with this device was not successful. Therefore, we introduced the SpiderFX ${ }^{\mathrm{TM}}$ Embolic Protection Device (Covidien, ev3 Endovascular, Inc., Plymouth, MN, USA) with $5.0 \mathrm{~mm}$ filter, and with expanded device we removed thrombi from the artery. Finally, we implanted the 5.0/28 mm PK Papyrus covered stent (Biotronik, Berlin, Germany) into the renal artery. The stent was expanded under the pressure of 16 atm. Implantation of this stent resulted in good revascularization of right kidney. After endovascular procedure clinical status of this patient steadily improved, biochemical parameters normalized and at follow-up 6 months after the procedure he no longer required hemodialysis. 


\subsection{Patient3}

A 52-year-old female patient was admitted to nephrological department due to acute renal failure, which required hemodialysis. CT angiography revealed occlusion of abdominal aorta just below origin of the superior mesenteric artery and occlusion of both renal arteries. There were also radiologic features of cirrhosis of the right kidney, while the left kidney was normally sized. Distal part of the left renal artery was patent and was filled with contrast from collateral circulation. Echocardiographic examination demonstrated significantly low ejection fraction $(30 \%)$, still with no signs of valvular dysfunction. In addition, preprocedural course was complicated by heparin-induced thrombocytopenia, which was successfully managed with fondaparinux. Considering the high risk of open surgical revascularisation we decided to attempt an endovascular repair of occluded abdominal aorta and the left renal artery using the chimney technique.

\subsubsection{Intervention}

Through the brachial access, using the $6 \mathrm{~F} / 90 \mathrm{~cm}$ Destination ${ }^{\circledR}$ guiding sheath (Terumo, Tokyo, Japan) and $125 \mathrm{~cm}$ long $5 \mathrm{~F}$ Vertebral hydrophilic diagnostic catheter Impress ${ }^{\circledR}$ (Merit Medical Systems Inc., South Jordan, UT, USA) we cannulated abdominal aorta up to the level of occlusion. Contrast injected at this level revealed that distal part of the left renal artery, about $4 \mathrm{~cm}$ from aorta, was patent received blood supply from collateral circulation. Using the $260 \mathrm{~cm}$ long hydrophilic guidewire AqWire $^{\mathrm{TM}}$ (Covidien, ev3 Endovascular, Inc., Plymouth, MN, USA) we navigated through occluded distal part of the aorta. Then, under fluoroscopic control, we introduced the 7F/45 $\mathrm{cm}$ Destination $^{\circledR}$ guiding sheath (Terumo, Tokyo, Japan) into the left femoral artery and using the loop we captured the guidewire that has been introduced from aorta. Since the origin of the left renal artery was not seen, we dilated the aorta in the area where such an origin should be located with $3.0 / 40 \mathrm{~mm}$ and $6.0 / 40 \mathrm{~mm}$ angioplastic balloons, which resulted in visualization of proximal portion of the left renal artery. With the use of V-14 ControlWireTM (Boston Scientific, Natick, MA, USA) hydrophilic-coated guidewire we navigated through occluded segment of this artery. Afterwards through right brachial access we cannulated the superior mesenteric artery and from left brachial access - the left renal artery. Then, using the chimney technique and simultaneous expansion of stents, we implanted $5 \times 38 \mathrm{~mm}$ BeGraft covered stent (Bentley InnoMed, Hechingen, Germany) in the superior mesenteric artery, the same stent in the left renal artery and a bigger 10x58 mm BeGraft stent in abdominal aorta. In order to cover entire stenosis of the abdominal aorta, we implanted another 10x58 mm BeGraft stent in the distal part of occlusion. Final angiographic result of the procedure was good, with restored flow to renal parenchyma of the left kidney. After endovascular repair biochemical renal parameters normalized and normal diuresis was restored.

\section{DISCUSSION}

Percutaneous revascularization of acutely occluded RA becomes a new therapeutic option for the treatment of renal failure caused by ischemia of the kidneys [1-6]. Such an endovascular management is technically feasible and can restore diuresis even in severely compromised patients and after relatively long period of renal ischemia. Yet, it remains unclear if such patients should be treated endovascularly, or instead they should be managed with life-long hemodialysis. We suggest that endovascular treatment should be considered in high risk patients who need an additional endovascular repair.

\section{REFERENCES}

[1] Silverberg D, Menes T, Rimon U, et al. Acute renal artery occlusion: presentation, treatment and outcome. J Vasc Surg 2016; 64: 1026-32.

[2] Latacz P, Rudnik A, Gutowska A, et al. Percutaneous angioplasty of the left renal artery in a patient with acute infarction of the left kidney with persistent occlusion of the right renal artery treated with angiotensin converting enzyme inhibitor. Kardiol Pol 2011; 69: 702-5.

[3] Kanamori H, Toma M, Fukatsu A., Improvement of renal function after opening occluded atherosclerotic renal arteries. J Invasive Cardiol 2009; 21: E171-4.

[4] Hedayati N, Lin PH, Lumsden $\mathrm{AB}$, et al. Prolonged renal artery occlusion after endovascular aneurysm repair: endovascular rescue and renal function salvage. J Vasc Surg 2008; 47: 446-9.

[5] Jongkind V, Kievit JK, Wiersema AM, Recovery of renal function after prolonged anuria in acute suprarenal aortic occlusion. Ann 
Vasc Surg 2016; 30: 307.e11-307.e14.

[6] Latacz P, Simka M, Krzanowski M, et al. Composed revascularization techniques for rescue endovascular management of acute occlusion of abdominal aorta and renal arteries. Pol Arch Intern Med 2018; 128: 319-21.

Citation: Pawel Latacz, Marian Simka, Marcin Krzanowski, Joanna Dolipska, Władysław Sułowicz. Endovascular Rescue Treatment for Occlusion of Renal Arteries Resulting in Acute Renal Failure. ARC Journal of Cardiology. 2018; 4(2) 1-4. doi: dx.doi.org/11.20431/2455-5991.0402001.

Copyright: (C) 2018Authors. This is an open-access article distributed under the terms of the Creative Commons Attribution License, which permits unrestricted use, distribution, and reproduction in any medium, provided the original author and source are credited. 